\title{
O Estado da Arte sobre o Enfoque CTS nas Revistas da Rede Nacional de Educação Profissional e Tecnológica
}

\author{
Pedro Victor de Araújo Sales* (Discente do curso técnico integrado em Mecânica - IFRN/Mossoró) \\ Ítalo de Sousa Marinheiro; (Discente do curso técnico integrado em Mecânica- IFRN/Mossoró) \\ Riston Alex Martins; (Discente do curso técnico integrado em Edificações- IFRN/Mossoró) \\ Albino Oliveira Nunes; (Professor de Química - IFRN/Mossoró) \\ Denilson Antonio Maia da Silva; (Professor de Química - IFRN/Mossoró) \\ * ítalo.sousa13@gmail.com
}

\begin{abstract}
resumo:
A educação para a cidadania é um dos grandes objetivos que se impõe aos sistemas educativos internacionais, e em especial para a educação brasileira. Nesse contexto, o enfoque Ciência, Tecnologia e Sociedade (CTS) surge como uma alternativa viável para a promoção da cidadania no ensino de ciências e tecnologia. Assim, este artigo tem como objetivo mapear a pesquisa sobre o enfoque CTS em revistas de instituições da Rede Nacional de Educação Profissional e Tecnológica (RNEPT). Para tanto, fez-se um levantamento de todos os artigos científicos publicados nas revistas científicas dos IF's, CEFET's e Universidade Tecnológica (UT), a partir dos sites respectivos de cada revista, que apresentam artigos sobre o tema. Ao final, conclui-se que o enfoque CTS está presente em poucos periódicos do Brasil e que, consequentemente, há uma lacuna quanto a pesquisas sobre as possibilidades do uso desse enfoque na educação profissional.
\end{abstract}

pallavras-chave:

CTS, Ensino, Estado da Arte, Rede de Educação Profissional. 
Espaço reservado para organização do congresso.

\section{Introdução}

O termo CTS trata da inter-relação entre a Ciência e Tecnologia com a sociedade, visto que os três campos, de forma natural, são propensos a se desenvolverem de forma individual e independente. Sua aplicação na matriz da sociedade, educação, possibilita o desenvolvimento do pensamento crítico do cidadão, tornando-o capaz de reagir de forma ética e responsável perante as informações que chegam a ele.

Sua abordagem no ensino/aprendizagem em qualquer nível de ensino ou conteúdo, agrega valores tanto ao conteúdo ou disciplina abordada quanto a quem a estuda, trazendo uma visão mais abrangente que envolva mais aspectos e perspectivas sobre um determinado assunto. O presente artigo, de caráter bibliográfico, possibilitará ao leitor uma visão de como a temática CTS é abordada nas revistas científicas dos IF's, CEFET's e UT levando à reflexão do quanto essa temática está presente e se faz relevante na esfera acadêmica.

\section{Material e métodos}

A metodologia do trabalho seguiu as seguintes etapas:

1- Levantamento de todos os periódicos publicados pelas instituições da Rede Nacional de Educação, Profissional e Tecnológica - RNEPT;

2- Escolha dos periódicos que publicam artigos nas áreas de ensino, educação e interdisciplinar;

3- Seleção de artigos que versavam sobre o enfoque CTS;

A partir do levantamento, foram encontrados 61 periódicos científicos, que abordam variadas áreas temáticas, distribuídos por todo o país ligados às instituições da rede federal de educação profissional e tecnológica. Dessas 61, 31 apresentavam artigos com conteúdo referente ao enfoque CTS/CTSA, ensino de química e atitudes no ensino de ciências. Feito isso, deu-se início à seleção dos artigos e a distribuição para os três integrantes do grupo de pesquisa. Tendo em vista que este trabalho faz parte de um projeto maior, o recorte aqui feito foi o de artigos que versaram sobre o enfoque CTS/CTSA. Ao todo foram encontrados 23 artigos com essa temática.

Com o objetivo de mapear os estudos e pesquisas científicas realizados nas instituições da Rede Nacional de Educação Profissional e Tecnológica (RNEPT) envolvendo aspectos do enfoque CTS e Ciência, Tecnologia, Sociedade e Ambiente (CTSA), fez-se um levantamento, no mês de agosto de 2015, de todos os artigos científicos publicados nas revistas científicas dos IFs, CEFET's e Universidade Tecnológica do período de 1994 até agosto de 2015, a partir dos respectivos sites de cada revista.

\section{Resultados e discussão}

Nas etapas em que o levantamento foi desenvolvido, foram obtidos 4012 artigos. Desses, 23 apresentavam conteúdo com aspectos CTS ou CTSA, ou seja, compõem o objeto dessa pesquisa, sendo sete destes artigos da revista Educação \& Tecnologia (CEFET-MG) entre o período de 2000 e 2014, seis da revista Tecnologia \& Cultura entre o period de 2008 e 2011, quatro da revista HOLOS (IFRN) nos anos 2010 e 2014, três da revista Debates Educação Científica e Tecnológica (DECT IFES) entre o período de 2013 e 2014, um da revista eletrônica Sala de Aula em Foco (IFES) em 2012, um da revista ciências \& Ideias (IFRJ) em 2014 e um da revista ACTA Tecnológica (IFMA) em 2011. 
Tabela 1 - Revistas selecionadas e seus respectivos Qualis.

\begin{tabular}{|c|c|c|c|c|}
\hline Revista & Ensino & Educação & Interdisciplinar & Qnt. de artigos \\
\hline Educação \& Tecnologia & B2 & B4 & B3 & 7 \\
\hline Tecnologia \& Cultura & B2 & B5 & B3 & 6 \\
\hline HOLOS & B5 & B2 & B2 & 4 \\
\hline DECT & B2 & $\mathrm{C}$ & - & 3 \\
\hline Sala de Aula em Foco & B3 & $\mathrm{C}$ & - & 1 \\
\hline Ciências \& Ideias & B1 & B5 & - & 1 \\
\hline ACTA Tecnológica & B4 & $\mathrm{C}$ & B5 & 1 \\
\hline
\end{tabular}

Fonte: dados da pesquisa (2015).

A partir seleção dos artigos selecionados, conforme a metodologia escolhida, foi possível classificar e assim, indicar qual instituição está mais presente na publicação de artigos relacionados ao enfoque CTS, como segue a tabela a seguir:

Tabela 2 - Relação dos artigos científicos com suas respectivas revistas, instituições e anos de publicação.

Título

Los estudios CTS - una crítica marxista a los contructivistas y a los críticos (A1)

A importância do enfoque CTS na graduação de Educação\&Tecnologia engenheiros (A2)

Tendências temáticas dos trabalhos em Física apresentados no II Seminário Ibero-Americano de CTS (II SIACTS-EC) (A3)

Aspectos do enfoque CTS no mapeamento das produções acadêmicas sobre a educação profissional de nível técnico (A4)

Temas sociocientíficos (cachaça) em aulas práticas de química na educação profissional uma abordagem CTS (A5)

Geração de energia elétrica - uma abordagem CTS na disciplina de Física Experimental (A6)

Ciência, tecnologia e sociedade (CTS) - O lugar do ensino de geografia (A7)

Projeto matrizes energéticas - Temática para o ensino de ciências a partir da abordagem CTSA (A8)

Alfabetização científica com enfoque CTSA: produção de um jornal da ciência no Ensino Médio público (A9)

As relações CTSA na Licenciatura em Química: uma proposta de recurso de didático voltado ao sertão nordestino (A10)

A interdisciplinaridade por meio da pedagogia de projetos: uma análise do projeto "Horta Escolar: aprenda cultivando ortaliças” numa perspectiva CTSA (A11)

Ideologia da certeza matemática - contribuições reflexivas do enfoque CTS (A12)

Atitudes e crenças sobre as relações CTSA de estudantes do curso de Edificações na modalidade EJA: uma análise
Revista

Instituição

Educação \&Tecnologia

CEFET-MG

CEFET-MG

Educação\&Tecnologia

CEFET-MG

Educação\&Tecnologia

CEFET-MG

Educação\&Tecnologia

CEFET-MG

Educação\&Tecnologia

CEFET-MG

Educação\&Tecnologia

CEFET-MG

Sala de Aula em Foco

IFES

Debates em Educação

Ciência e Tecnologia

Debates em Educação

Ciência e Tecnologia

IFES

IFES

Debates em Educação

Ciência e Tecnologia

Ciências\&Ideias

IFRJ

HOLOS

IFRN 
por períodos (A13)

Discussão e debate de questões CTS por alunos do último

HOLOS

IFRN

ano de um curso de Licenciatura em Química: definições de ciência e tecnologia (A14)

O ensino de acústica nos livros didáticos de física recomendados pelo PNLEM: análise das ligações entre a física e o mundo do som e da música (A15)

Utilização de mapa conceitual como ferramenta de análise de trabalhos científicos (A16)

Bases epistemológicas subjacentes ao enfoque CTS no ensino de química (A17)

Perspectivas de atividades experimentais em biologia, Cultura \& Tecnologia

IFRN

considerando o enfoque CTS (A18)

O enfoque CTS - Ciência, Tecnologia e Sociedade e seus Cultura \& Tecnologia

IFRN

impactos no ensino (A19)

Concepções de professores en formación sobre naturalesa Cultura \& Tecnologia

\section{IFMA}

CEFET-RJ

CEFET-RJ

de la ciencia y la tecnología (A20)

Ciencia, tecnología, Sociedad y Educación - una Cultura \& Tecnologia

CEFET-RJ

perspectiva de gênero (A21)

Aproximações sobre a organisação do conhecimento

científico nas propostas pedagógicas baseadas em Paulo

Freire e CTS no ensino de ciências (A22)

O que é CTS, afinal, na Educação Tecnológica (A23)

Cultura \& Tecnologia

CEFET-RJ

Cultura \& Tecnologia

CEFET-RJ

Fonte: dados da pesquisa (2015).

A partir da leitura do Quadro 2, pode observar-se que as revistas Educação \& Tecnologia, Tecnologia \& Cultura e HOLOS se fizeram mais presentes na publicação de artigos científicos que versem o enfoque CTS. Essa informação essa que possibilita uma melhor compreensão de que apesar de sua importância em outros países e contextos esse enfoque permanece pouco explorado na educação profissional brasileira. Havendo, no entanto, alguns focos a partir dos quais a pesquisa com esse enfoque parece se estabelecer.

Abaixo descrevemos as revistas nas quais foi encontrado maior número de artigos e seus perfis de publicações.

\subsection{Revista Educação \& Tecnologia}

A revista Educação \& Tecnologia é um periódico quadrimestral de divulgação científica mantido pelo Centro Federal de Educação Tecnológica de Minas Gerais - CEFET-MG desde 1994.

O propósito deste veículo é publicar textos relevantes para as discussões que inter-relacionam educação e tecnologia, na forma de relatos de pesquisa básica ou aplicada, do desenvolvimento de processo ou produto, da experiência pedagógica ou de projetos implementados de ensino ou de intervenção social e educacional.

\subsection{Revista Tecnologia \& Cultura}

A Revista Tecnologia \& Cultura é um periódico de divulgação científica do Centro Federal de Educação Tecnológica Celso Suckow da Fonseca, cujo papel é de agente de socialização do conhecimento produzido no CEFET/RJ e na comunidade acadêmica. Nesse sentido, a revista possui seu escopo editorial de caráter abrangente, multidisciplinar, aberto a contribuições de docentes, pesquisadores, dentre outros profissionais da área de Tecnologia. A publicação de seus textos são voltados para as subáreas de Tecnologia \& Sociedade, Tecnologia \& Gestão e Tecnologia \& Inovação.

\subsection{Revista HOLOS}

A HOLOS é uma publicação online do Instituto Federal do Rio Grande do Norte (IFRN) que tem como objetivo publicar artigos que contribuam para o estudo de temas interdisciplinares. O periódico recebe contribuições em português, inglês e espanhol desde 2004. 


\section{Análise do perfil das publicações}

No artigo Geração de energia elétrica - uma abordagem CTS na disciplina de Física Experimental (A6), os autores Marchezini e Teixeira desenvolvem experimentos de física que envolvem produção de energia elétrica com alunos de Química Tecnológica, analisando suas competências e habilidades dentro de uma perspectiva CTS. Simultaneamente, investigando as contribuições do enfoque no aprimoramento da disciplina até concluir que a inclusão dessa abordagem acrescenta valores ao ensino experimental de Física.

No artigo As relações CTSA na Licenciatura em Química: uma proposta de recurso de didático voltado ao sertão nordestino (A10), os autores Nunes, Dantas e Souza (2013) descrevem a elaboração de recurso didático sobre as relações CTSA voltada ao curso de licenciatura em química e é apresentado um tema local para o ensino de química dentro de uma perspectiva CTSA: O Rio Apodi Mossoró.

Nesse sentido, a tríade Ciência, Tecnologia e Sociedade (CTS) estuda basicamente a interrelação dos três segmentos e busca entender os aspectos sociais do desenvolvimento tecnocientífico tanto nos benefícios como nas consequências sociais e ambientais. (PINHEIRO et al., 2009).

Tabela 3 - Relação de nível de ensino, conteúdo e caráter (teórico, bibliográfico ou prático) dos artigos científicos abordados.

\begin{tabular}{llll} 
Título & Nível de ensino & Conteúdo & Aspecto \\
\hline A1 & NE & Geral & Teórico \\
A2 & NS & Engenharia & Prático \\
A3 & NS & Física & Bibliográfico \\
A4 & NT & Geral & Bibliográfico \\
A5 & NT & Química & Prático \\
A6 & NE & Física & Teórico \\
A7 & NE & Geografia & Teórico \\
A8 & NM & Produção de energia & Prático \\
A9 & NM & Geral & Prático \\
A10 & NS & Química & Prático \\
A11 & NE & Geral & Prático \\
A12 & NM & Matemática & Teórico \\
A13 & NT & Geral & Prático \\
A14 & NS & Química & Prático \\
A15 & NM & Multidisciplinar & Bibliográfico \\
A16 & NE & Geral & Teórico \\
A17 & NE & Química & Teórico \\
A18 & NM & Biologia & Teórico \\
A19 & NE & Geral & Teórico \\
A20 & NM & Geral & Prático \\
A21 & NM & Geral & Prático \\
A22 & NE & Geral & Teórico \\
A23 & NE & Geral & Teórico \\
Fo: & Na &
\end{tabular}

Fonte: dados da pesquisa (2015).

Legenda: NT = Nível Técnico; NS = Nível Superior; NE = Não específico; NM = Nível Médio;

Após a observação da tabela anterior, é possível perceber uma baixa incidência de artigos com conteúdo voltado à área da matemática e física, ocorrência essa contrária nas área de química e não específica, que representam aproximadamente $17 \%$ e $47 \%$, respectivamente, do total dos artigos encontrados. 
Outra observação que pode ser feita, é predominância do caráter prático nos artigos, o que indica uma aplicação, de fato, do enfoque CTS nos diversos objetos acadêmicos possíveis, seja em algum projeto, no ensino/aprendizagem, etc. Quanto ao teórico, que está presente em aproximadamente $43 \%$ dos artigos, trata da conscientização, geralmente, da esfera acadêmica sobre a importância de sua inclusão no caráter do cidadão e consequentemente da sociedade como um todo. Esse resultado torna-se relevante à medida que diferencia os trabalhos publicados nessas revistas de outros veículos de divulgação da pesquisa em ensino de ciências nas quais predominam trabalhos teóricos sobre o tema (STRIEDER, 2012).

\section{CONCLUSÕES}

Os resultados dos estudos nos permitem afirmar que o enfoque CTS ainda se mostra pouco abordado nos periódicos científicos dos IF's, visto que de 4012 artigos científicos, dentre todos os periódicos desde 1994 até atualmente em 2015, apenas 10 abordam o enfoque CTS em sua temática.

Tal problema exige atenção, pois a nível mundial esse enfoque tem sido incorporado aos mais diversos níveis educacionais, sobretudo na educação tecnológica. Nesse contexto, a pesquisa ressalta a importância de se promover investigações com o enfoque CTS no âmbito da educação profissional e tecnológica do Brasil.

\section{State of the Art on the Focus STS in Magazines of the National Network of Professional Education and Technology}

Albstract:Education for citizenship is one of the great objectives that imposes on international education systems, especially for Brazilian education. In this context, the focus Science, Technology and Society (STS) emerges as a viable alternative for promoting citizenship in science education and technology. Thus, this article aims to map the research on the CTS approach in magazines institutions of the National Network of Vocational and Technological Education (NNVTE). Therefore, there was a survey of all scientific articles published in scientific journals of IF's, CEFET's and Technological University (UT), from the respective websites of each journal, presenting articles on the subject. Finally, it is concluded that the CTS approach is present in a few journals from Brazil and that, consequently, there is a gap as the research on the possibilities of using this approach in education.

Keywords: State of the Art; Science-Technology-Society, STS, Professional Education

\section{Referências bibliográfica}

ALMEIDA, J. S. DE et al. A interdisciplinaridade por meio da pedagogia de projetos: uma análise do projeto "Horta Escolar: aprenda cultivando hortaliças " numa perspectiva CTSA. Debates em Educação Científica e Tecnológica, v. 4, n. 1, p. 179-191, 2014.

ARAÚJ O, M. S. T.; FORMENTON, R. Utilização de mapa conceitual como ferramenta de análise de trabalhos científicos. HOLOS, v. 1, p. 171-181, 2015.

ALONSO, Á. V.; MAS, M. A. M. Concepções de professores en formación sobre naturalesa de la ciencia y la tecnología.pdf. Tecnologia \& Cultura, n. 13, p. 18-28, 2008.

BAZZO, W. A.; PEREIRA, L. T. DO V. O que é CTS, afinal, na Educação Tecnológica.pdf. Tecnologia \& Cultura, n. 13, p. 46-53, 2008. 
CEREZO, J. A. L. Ciencia, Tecnología y Sociedad: el estado de la cuestión en Europa y Estados Unidos. Revista I beroamericana de Educación, v. 18, p. 41-68, 1998.

CHRISPINO, A. O enfoque CTS - Ciência, Tecnologia e Sociedade e seus impactos no ensino.pdf. Tecnologia \& Cultura, n. 13, p. 7-17, 2008.

CORRÊA, A.; ARAÚj O, M. Tendências temáticas dos trabalhos em Física apresentados no II Seminário Ibero-Americano de Ciência , Tecnologia e Sociedade ( II Resumo Introdução. Educação \& Tecnologia, v. 16, n. 2, p. 23-45, 2010.

CORRÊA, A.; ARAÚj O, M. Aspectos do enfoque Ciência-Tecnologia-Sociedade ( CTS ) no mapeamento das produções acadêmicas sobre a educação profissional de nível técnico no âmbito das instituições federais de educação tecnológica Resumo. Educação \& Tecnologia, v. 18, n. 3, p. 9-23, 2011.

GLAVICH, E. E. Los Estudios CTS: una crítica marxista a los constructivistas y a los críticos. Educação \& Tecnologia, v. 5, n. 1, p. 21-26, 2000.

KRUGER, J.; PREZILIUS, A.; LEITE, S. Alfabetização científica com enfoque CTSA: produção de um jornal da ciência no Ensino Médio público. Debates em Educação Científica e Tecnológica, v. 3, n. 2, p. 79-97, 2013.

LOBINO, M.; NETO, G. Projetos Matrizes energéticas: temática para o ensino de ciências a partir da abordagem CTSA. Sala de Aula em Foco, v. 01, n. 2, p. 31-38, 2012.

MARCHEZINI, R.; TEIXEIRA, M. Geração de energia elétrica: uma abordagem Ciência, Tecnologia e Sociedade na disciplina de Física Experimental 1. Educação \& Tecnologia, v. 19, n. 3, p. 46-58, 2014.

MATIAS, V. Ciência, tecnologia e sociedade: O lugar do ensino de geografia. Educação \& Tecnologia, v. 17, n. 3, p. 56-66, 2012.

MELO, T.; CHRISPINO, A. Ideologia da certeza matemática: contribuições reflexivas do enfoque CTS. Ciências \& I deias, v. 5, n. 2, p. 100-119, 2014.

MONTEI RO JÚNIOR, F. N.; CARVALHO, W. L. P. DE. O ensino de acústica nos livros didáticos de física recomendados pelo PNLEM: análise das ligações entre a física e o mundo do som e da música. HOLOS, v. 1, p. 137-154, 2011.

NUNES, A. O. et al. Atitudes e crenças sobre as relações CTSA de estudantes do curso de Edificações na modalidade EJ A: uma análise por períodos. HOLOS, v. 5, p. 244-254, 2010.

NUNES, A. O.; DANTAS, M.; SOUZA, L. DI. As relações CTSA na licenciatura em química: uma proposta de recurso didático. Debates em Educação Científica e Tecnológica, v. 03, n. 2, p. 3351, 2013.

PINTO, J. A.; MACIEL, M. D. Discussão e debate de questões CTS por alunos do último ano de um curso de Licenciatura em Química: definições de ciência e tecnologia. HOLOS, v. 01, p. 247-257, 2014.

PORRO, S. Ciencia, tecnología, Sociedad y Educación - una perspectiva de gênero. pdf. Tecnologia \& Cultura, n. 13, p. 29-36, 2008.

SANTOS, M.; AMARAL, C.; MACIEL, M. Temas sociocientíficos (cachaça) em aulas práticas de química na educação profissional: uma abordagem CTS. Educação \& Tecnologia, v. 16, n. 1, p. 37-48, 2011. 
SOUZA, F. L.; GONÇALVES, T. V. O. Bases epistemológicas subjacentes ao enfoque CTS no ensino de química. ACTA Tecnológica, v. 6, n. 2, p. 30-36, 2011.

SOUSA, C. M.; GOMES, G. F. A importância do enfoque CTS na graduação de engenheiros. Educação \& Tecnologia, v. 15, n. 2, p. 87-94, 2010.

TRÓPIA, G.; AMORIM, F.; MARTINS, M. C. Aproximações sobre a organisação do conhecimento científico nas propostas pedagógicas baseadas em Paulo Freire e CTS no ensino de ciências.pdf. Tecnologia \& Cultura, n. 13, p. 37-45, 2008.

VAZ, C. R. ;FAGUNDES, A. B.; PINHEIRO, N. A. M. O Surgimento da Ciência, Tecnologia e Sociedade ( CTS ) na Educação: Uma Revisão. I Simpósio Nacional de Ensino de Ciência e Tecnologia, v. Anais, n. Uma revisão, p. 98-116, 2009.

VILARDO, M. DE C. B.; MATOS, G. I.; AZEVEDO, M. Perspectivas de atividades experimentais em biologia, considerando o enfoque CTS. Tecnologia \& Cultura, n. 13, p. 7-16, 2011. 